Laser Chem., 1999, Vol. 19, pp. 169-172

Reprints available directly from the publisher Photocopying permitted by license only
(C) 1999 OPA (Overseas Publishers Association) N.V. Published by license under the Harwood Academic Publishers imprint, part of The Gordon and Breach Publishing Group.

\title{
TEMPERATURE EFFECTS OF EXCITATION LASER PULSES DURING STEP-SCAN FT-IR MEASUREMENTS
}

\author{
C. RÖDIG ${ }^{\mathrm{a}, *}$, H. GEORG ${ }^{\mathrm{a}}$, F. SIEBERT $^{\mathrm{a}}$, I. ROUSSO $^{\mathrm{b}}$ \\ and M. SHEVES ${ }^{\mathrm{b}}$ \\ ${ }^{a}$ Institut für Biophysikund Strahlenbiologie, Albert-Ludwigs-Universität, \\ Albertstrasse 23, 79104 Freiburg, Germany; \\ ${ }^{\mathrm{b}}$ Department of Organic Chemistry, The Weizmann Institute of Science, \\ Rehovot 76100, Israel
}

(Received 6 April 1997)

\begin{abstract}
Replacing the chromophore of bacteriorhodopsin with chemically modified retinal analogs which cannot isomerize, allows to measure directly side effects from the laser pulse used for sample excitation in step-scan FT-IR measurements. Comparison with static temperature difference spectra and temperature-jump experiments shows that the observed effects can mainly be attributed to heating of the sample by the laser pulse. The size of resulting spectral changes is compared to difference bands of the photoreaction.
\end{abstract}

Keywords: Step-scan FT-IR spectroscopy; bacteriorhodopsin; locked retinal analogs

Laser excitation is commonly used to drive cyclic reactions of biological systems in time-resolved infrared difference spectroscopy $[1,2]$. Our experimental setup for measurements with the FT-IR stepscan technique includes a frequency doubled Nd:YAG laser with $1.5 \mathrm{~mJ}$ pulse energy for this purpose. This laser pulse may in some cases not only drive the photoreaction, but also exert a considerable side effect on the sample. In the present work we analyzed the contribution of such side effects to the difference spectrum in the case of the photocycle of bacteriorhodopsin (BR). The first step in the

\footnotetext{
* Corresponding author.
} 
photocycle after light absorption is the isomerization of the chromophore from the all-trans to 13-cis configuration [3]. In order to suppress spectral changes arising from this and the following steps in the photocycle, we replaced the retinal chromophore by three different chemically modified retinals, which cannot isomerize upon light absorption (Fig. 1) [4].

We performed step-scan measurements with films of the three artificial pigments, which all show very similar difference bands in the amide I and amide II regions. All bands decay within a few hundreds of microseconds with identical kinetics. The band size is about 5 percent of the largest difference bands occuring in the photocycle of BR. Since in these measurements the photocycle is inhibited, we explain the observed spectral features by alterations of the protein environment of the chromophor due to a local temperature rise in the

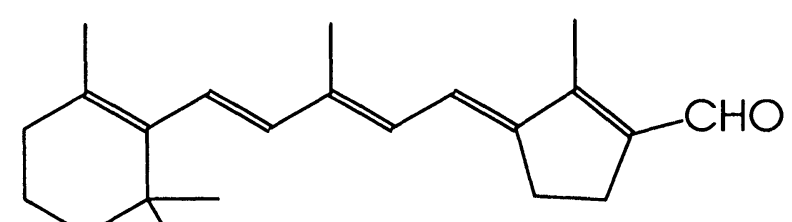

CHROMOPHORE I
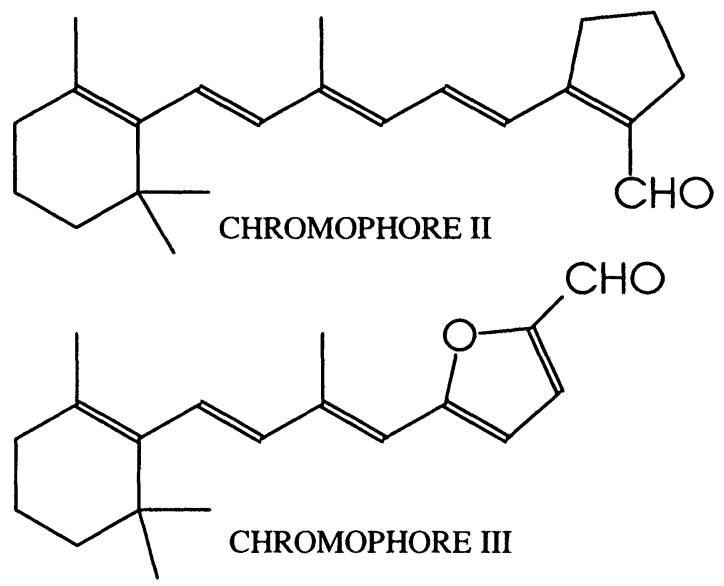

FIGURE 1 Retinal analogs used as chromophor in artificial pigments I, II and III. 


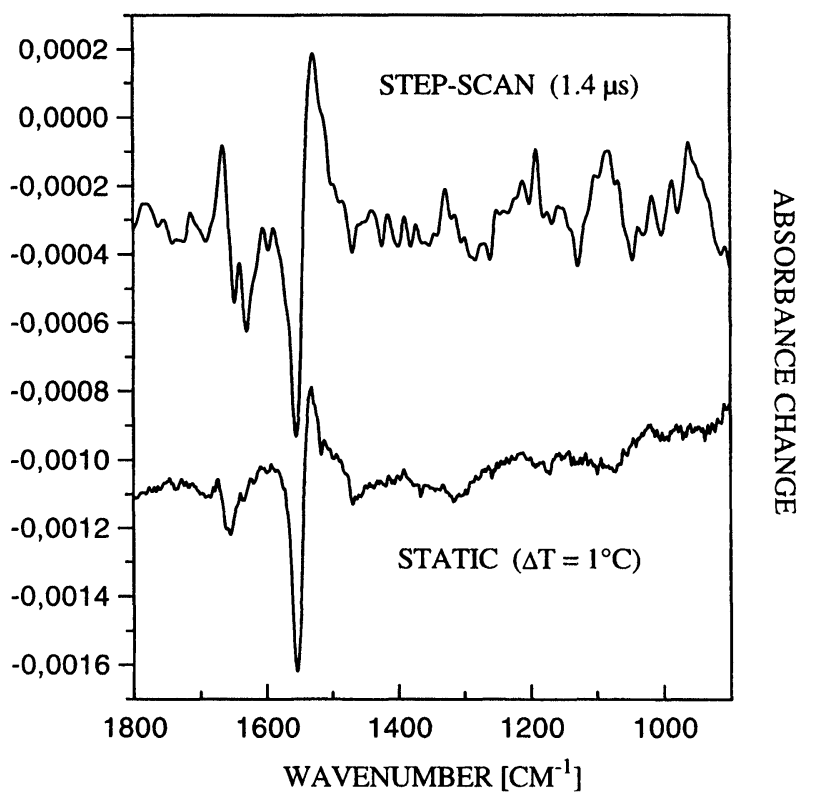

FIGURE 2 Comparison of difference spectrum obtained from time-resolved step-scan measurements and static temperature difference spectrum of artificial pigment I.

sample. This heating effects is mainly caused by release of thermal energy due to excess photon energy from the excitation laser pulse.

Static temperature difference spectra obtained from the same samples by varying the temperature in steps of $5^{\circ} \mathrm{C}$ reproduce the results obtained from step-scan measurements (Fig. 2). Additional evidence for the interpretation of the effect is obtained from time resolved temperature-jump experiments, where the sample is heated by an infrared laser pulse. Again very similar kinetic with the same sign of absorption change are observed at the corresponding spectral positions.

In conclusion we can estimate the temperature rise in the sample caused by the excitation laser pulse to be about $1^{\circ} \mathrm{C}$. The related spectral changes must be taken into account when interpreting timeresolved infrared difference spectra, especially when the spectral changes due to the photoreaction are small. 


\section{References}

[1] Uhmann, W., Becker, A., Taran, C. and Siebert, F. (1991). Appl Spectr., 45, 390.

[2] Hage, W., Kim, M., Frei, H. and Mathies, R. A. (1996). J. Phys. Chem., 100, 16026.

[3] Braiman, M. S. and Mathies, R. A. et al. (1980). Biochemisty, 19, 5421.

[4] Ottolenghi, M. and Sheves, M. (1989). J. Mem. Biol., 112, 193. 